\title{
Probabilistic Power Flow Prediction of Micro-grid Comprising Wind and Photovoltaic Generation
}

\author{
Song-Lin Zhou, Zeng-Liang Liu \\ Department of Electrical Engineering, \\ Tongling University, \\ Tongling, 244000, China \\ E-mail: zsl040908@163.com, lzl@tlu.edu.cn
}

\begin{abstract}
The power flow of micro-grid containing wind-light complementary system shows greater uncertainty due to it is affected by wind speed and irradiance. Therefore the prediction of probabilistic power flow is of great significance for micro-grid energy management. In the paper, on the basis of wind power and photovoltaic power prediction, a conditional joint probability prediction technique is put forward to predict probabilistic power flow considering the correlation between wind and irradiance. The prediction technique is not only consider the affection that wind speed and irradiance put on the power flow, but also consider the co-constrains between wind speed intervals and irradiance intervals at the prediction moment. The simulation results show that the probabilistic intervals achieved by the proposed prediction methods are narrower than unconditional joint probability prediction technique and of more reference value.
\end{abstract}

Keywords-micro-grid; probabilistic power flow; conditional joint probability prediction; probabilistic interval

\section{INTRODUCTION}

Micro-grid connects some distributed generation equipments such as fuel cell, micro gas turbine, photovoltaic generation and wind generation in a certain area to supply power to users [1-3]. Micro-grid can make full use of all kinds of generation equipments to alleviate the pressure of power supply, and improve the reliability and economy of power supply system. Therefore, carrying out the research on the micro-grid is of important practical significance [4].

Due to the geographical distribution, seasonal changes and day-night alternate, wind and solar energy are complementary to a certain extent. So setting up wind-solar generation complementary system in a micro-grid can make up for the weakness of independent solar or wind generation system. The strong intermittent and randomness of wind and irradiance put more complex influence on micro-grid which comprises wind and photovoltaic generation than that comprises independent wind or photovoltaic generation.

Although the power flow of a micro-grid can be predicted further based on the prediction of wind and photovoltaic generation, wind and photovoltaic generation prediction are usually of larger errors that result in more complicated predictive errors of power flow. Therefore, it is of practical significance to assess the risk of the predictive power flow by solving the probability distribution and confidence interval based on the evolution characteristics of wind and irradiance.
Some scholars have studied the probabilistic power flow of micro-grid and distribution network. The Newton method had been adopted to solve the three-phase power flow of islanding micro-grid in reference [5].

The randomness of wind generation, photovoltaic generation and load had been studied firstly and then the probabilistic models are set up respectively with half invariant method to calculate probabilistic power flow of distribution network in reference [6]. Yu Kun established the dynamic probability model of random variables such as wind speed, irradiance and load, and applied the approximate method to calculate the probabilistic power flow of area power grid [7].

The adverse impacts of wind speed and irradiance in micro-grid can be prevented by prediction of wind generation and photovoltaic generation. Due to the impacts which the wind speed and illumination put on each node voltage and branch power of micro-grid are not same, so the directly prediction of micro-grid power flow is able to provide a more direct and comprehensive information for energy management system of micro-grid.

Few researches related to the power flow prediction of micro-grid concluding distributed generations were carried out at present. Although reference [8] predicted the wind generation and calculated the probabilistic power flow of power system, it did not study the probability distribution and confidence interval of power flow at each prediction moment. Deterministic and probabilistic power flow in micro-grid are predicted in reference [9], but the author did not take into account the correlation between wind speed and irradiance when the micro-grid comprises wind and photovoltaic generation simultaneous.

As we known, the power flow of a micro-grid is a function of wind speed and irradiance, and their influences on power flow have certain correlation and mutual constraint. One of the difficulties in the research is how to calculate the probability distribution of wind speed and irradiance. Another difficulty is how to solve the conditional joint probability of power flow about wind speed and irradiance at the prediction moment.

For the first difficulty, reference [10] pointed out that wind speed obeys weibull distribution and irradiance obeys normal distribution. In fact, the probability distribution of wind speed and irradiance are often different during different time even at same place. So using uniform distribution function to describe wind speed or irradiance is not 
appropriate. For the second difficulty, few researches related to the topic are carried out at present all over the world.

In view of the present researches, the paper analyzed the Markov characters of wind speed and irradiance firstly, and then calculated the conditional joint probability distribution (CJPD) and the confidence intervals of micro-grid power flow at the prediction moment by Markov process model and Latin hypercube sampling.

The proposed CJPD technology considering the mutual constraints between wind speed intervals and irradiance intervals at predicted moment can effectively assess the risk of predictions of power flow. The probabilistic prediction results achieved by CJPD are more in line with the actual and of more significance for the energy scheduling of micro-grid than that achieved by unconditional joint probability distribution (UJPD) without considering the correlation between two random variables.

\section{POWER FLOW PREDICTION OF MICRO-GRID}

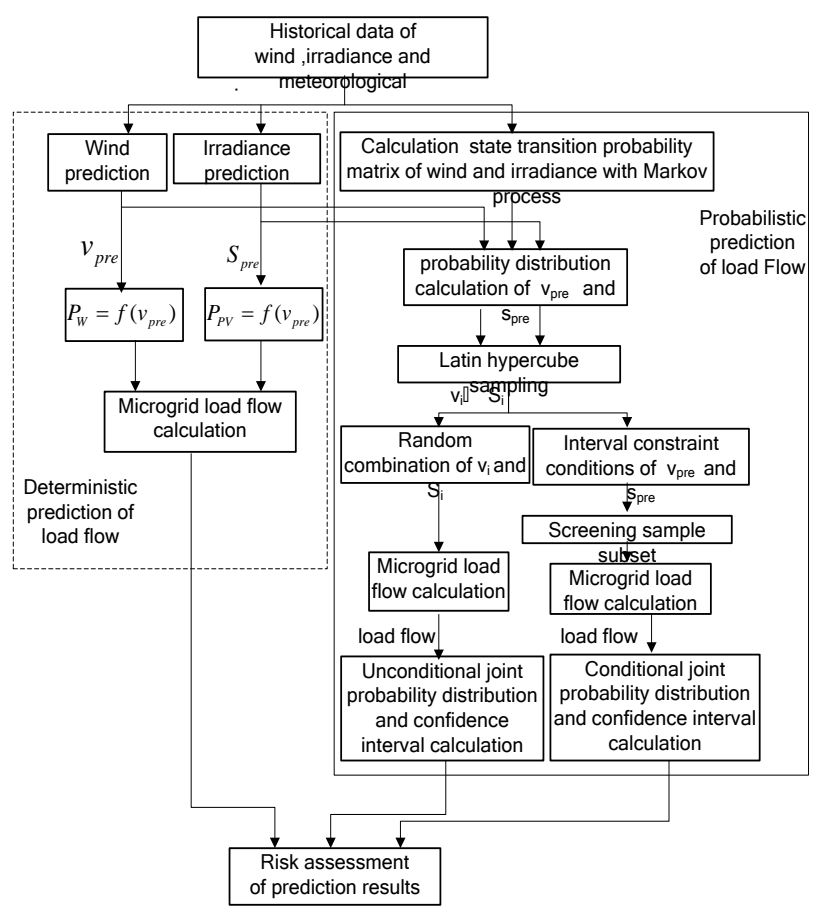

Figure 1. Power flow prediction procedure diagram.

Figure 1 is the power flow prediction procedure that consists of deterministic prediction and probabilistic prediction.

(a)Deterministic power flow prediction

The predictions of wind speed and irradiance are performed firstly according to the historical wind speed, irradiance and other meteorological data. After that, the predicted wind speed and irradiance are respectively transformed to wind generation and photovoltaic generation. Finally, these predicted wind and photovoltaic generation are substituted into power flow equations of the micro-grid to predict deterministic power flow.
(b)Probabilistic power flow prediction

There are two probabilistic power flow prediction methods are proposed in the paper. One is the unconditional joint probability distribution prediction which is performed by independently sampling wind speed and irradiance. Another is the conditional joint probability distribution prediction which considering the mutual constraints between wind speed intervals and irradiance intervals at the predict moment. The state transition probability matrix of wind speed and irradiance is solved by markov chain model according to the historical data of wind speed and irradiance intensity. Their state transition probability matrix and the prediction values are combined to solve the probability distribution functions (PDF) of wind speed and irradiance. According to the PDF, the samples of wind speed and irradiance are achieved by Latin hypercube sampling to calculate CJPD, UJPD and confidence intervals of power flow.

Deterministic power flow prediction values usually comprise certain risk due to their predicted errors. Probabilistic power flow prediction is helpful to assess the risk in micro-grid energy manager.

\section{DETERMINISTIC POWER FLOW PREDICTION OF MICRO-GRID}

\section{A. Prediction of Wind Speed and Wind Generation}

In the following research of probabilistic power flow, the predicted wind speed will be used to solve the PDF of wind speed, so we adopt the indirectly prediction method of wind power. We predict wind speed using ridgelet neural network based on historical meteorological data firstly [11], and then get the wind power according to formula (1).

$$
P_{w}= \begin{cases}0, & v \leq v_{c i} \\ k_{1} v+k_{2}, & v_{c i}<v \leq v_{r} \\ P_{r}, & v_{r}<v \leq v_{c o} \\ 0, & v>v_{c o}\end{cases}
$$

In formula (1), $P_{r}$ is rated power; $v_{r}$ is rated wind speed; $v$ is wind speed; $v_{c i}$ is cut-in speed; $v_{c o}$ is cut-out speed; $k_{1}=P_{r} /\left(v_{r}-v_{c i}\right) ; k_{2}=-k_{1} \cdot v_{c i}$.

B. Prediction of Irradiance and Photovoltaic Generation

There are lots of influence factors related to the prediction of photovoltaic generation such as irradiance, temperature, efficiency of PV array, installation angle, atmospheric pressure and types of weather, etc. For photovoltaic generation prediction, there are two factors should be concerned.

(a) Micro-grid is usually developed just in recent years, which results in that the long-term historic data of photovoltaic generation is lack.

(b) The irradiance should be predicted to calculate the state transition probability in following probabilistic power 
flow prediction. So we predict the irradiance by least squares support vector machine (LSSVM ) based on similar day method according to the weather condition firstly [12], and then predict photovoltaic generation by photoelectric conversion relations by following formulas[13].

$$
\begin{gathered}
P_{P V}=\eta A S \eta_{m p p t} \eta_{i n v} \\
Q_{P V}=P_{P V} \cdot \tan \varphi
\end{gathered}
$$

Where $P_{P V}, Q_{P V}$ are active power and reactive of the PV system, $\eta$ is efficiency of PV array, $A$ is effective area of PV array, $\mathrm{S}$ is irradiance, $\eta_{m p p t}$ is tracking efficiency of maximum power point, $\eta_{i n v}$ is efficiency of the inverter, $\varphi$ is inverter power factor angle of photovoltaic power station.

\section{Deterministic power flow prediction}

Deterministic power flow prediction is achieved by substituting the predicted wind power and photovoltaic power into following power flow equation.

$$
\begin{gathered}
\dot{U}_{i}=e_{i}+j f_{i} \\
P_{i}=e_{i} \sum_{j=1}^{n}\left(G_{i j} e_{j}-B_{i j} f_{j}\right)+f_{i} \sum_{j=1}^{n}\left(G_{i j} f_{j}+B_{i j} e_{j}\right) \\
Q_{i}=f_{i} \sum_{j=1}^{n}\left(G_{i j} e_{j}-B_{i j} f_{j}\right)-e_{i} \sum_{j=1}^{n}\left(G_{i j} f_{j}+B_{i j} e_{j}\right)
\end{gathered}
$$

Where, $\dot{U}_{i}, e_{i}, f_{i}$ separately denote node voltage, real part of node voltage and imaginary part of node voltage, $G_{i j}, B_{i j}$ denote conductance and susceptance, $P_{i}, Q_{i}$ denote active power and reactive power.

\section{PROBABILITY DISTRIBUTION PREDICTION OF WIND SPEED AND IRRADIANCE}

A. Markov property of wind speed and irradiance time series

Markov prediction can reveal the future trend of a system based on the state transition probability. The state transition probability is able to reflect the inherent regularity that system changes from a status to anther status. Reference [14-15] has analyzed the Markov property of wind speed and irradiance time series. Statistics $\chi^{2}$ is usually used to test the Markov property of discrete sequence [16]. Statistics $\chi^{2}$ described as formula 7 .

$$
\chi^{2}=2 \sum_{j=1}^{m} \sum_{k=1}^{m} N_{j k}\left|\ln \frac{p_{j k}}{p_{k}}\right|
$$

Assume that formula (7) obey $\chi^{2}$ distribution whose freedom degree is $(n-1)^{2}$. For a confidence level $\alpha$, we look up the value of $\chi_{\alpha}^{2}\left((n-1)^{2}\right)$ from statistical table. When $\chi^{2}>\chi_{\alpha}^{2}\left((n-1)^{2}\right)$, we judge that the time series behave Markov property. In the paper, we assume that $\alpha=0.05$ and freedom degree equal to 4 . For wind speed time series, we get $\chi_{0.05}^{2}\left((4-1)^{2}\right)=0.711, \chi^{2}=9.463$. For irradiance time series, we get $\chi_{0.05}^{2}\left((4-1)^{2}\right)=0.207$, $\chi^{2}=47.544$. It can prove that the wind and irradiance time series are of Markov property.

\section{B. Probability distribution of wind speed and irradiance}

The probability distribution of wind speed and irradiance at predicted moment are easily calculated by the state transfer probability matrix. Taking wind speed as an example, the historical wind speed data are divided into $m$ intervals that represent $m$ states. The original states $\mathbf{p}_{\mathbf{n}}$ is defined as:

$$
\mathbf{P}_{\mathbf{n}}=\left[\begin{array}{c}
P\left(V_{n}=v_{1}\right) \\
P\left(V_{n}=v_{2}\right) \\
\vdots \\
P\left(V_{n}=v_{m}\right)
\end{array}\right], n=0,1,2 \cdots
$$

Where $\mathbf{p}_{\mathbf{n}}$ is the column vector including all state probabilities of wind speed at the $n$ moment, $\mathrm{P}($.$) is event$ probability, $V_{\mathrm{n}}$ is random variable, $v_{i}(i=1, \cdots, m)$ is the state of a system. Assuming $p_{j k}=P\left(V_{n+1}=v_{k} \mid V_{n}=v_{j}\right)$ is the state transfer probability which denotes the probability that wind speed transforms from state $v_{j}$ to state $v_{k}$. The $p_{j k}$ can be achieved as following two steps.

(a) Assuming that the length of wind interval is $\Delta v$, wind speed range is $\left[v_{l}, v_{h}\right], i=1, \cdots, m$, thus the intervals given by

$$
\begin{gathered}
D_{i}=\left[v_{l}+(i-1) \cdot \Delta v, \quad v_{l}+i \cdot \Delta v\right] \\
m=\left[\left(v_{h}-v_{l}\right) / \Delta v\right]+1
\end{gathered}
$$

(b) Determining which interval the historical wind speed belongs to and calculating the transfer frequency matrix $N$ by counting the number that wind speed evolution from interval $D_{\mathrm{j}}$ to $D_{\mathrm{k}}$.

$$
N=\left[\begin{array}{lccc}
N_{11} & N_{12} & \cdots & N_{1 m} \\
N_{21} & N_{22} & \cdots & N_{2 m} \\
\vdots & \vdots & & \vdots \\
N_{m 1} & N_{m 2} & \cdots & N_{m m}
\end{array}\right]
$$


Assuming that $p_{k}$ is the transition probability from the other state to $k$ state, $\quad p_{j k}$ is an element of the state transition probability matrix.

$$
\begin{gathered}
p_{k}=\frac{\sum_{j=1}^{m} N_{j k}}{\sum_{j=1}^{m} \sum_{k=1}^{m} N_{j k}} \\
p_{j k}=\frac{N_{j k}}{\sum_{k=1}^{m} N_{j k}}
\end{gathered}
$$

The state transfer probability matrix written as

$$
\mathbf{P}=\left[\mathbf{p}_{\mathbf{j k}}\right]=\left[\begin{array}{cccc}
P_{11} & P_{12} & \cdots & P_{1 N} \\
P_{21} & P_{22} & \cdots & P_{2 N} \\
\vdots & \vdots & & \vdots \\
P_{N 1} & P_{N 2} & \cdots & P_{N N}
\end{array}\right]
$$

The wind speed PDF of all state at all moment can be calculated by

$$
\mathbf{P}_{n+1}=\mathbf{P} \cdot \mathbf{P}_{\mathbf{n}}
$$

Where $\mathbf{p}_{\mathbf{n}}$ is the original states, $\mathbf{p}$ is the state transfer probability matrix.

Assuming that $v(t)$ is the measured wind speed at time $t$, $v^{*}(t+d)$ is the predicted wind speed at time $t+d$. Then we calculate the transfer probability from state $v(t)$ to other states according to formula (15) on the base of the known wind speed $v(t)$. Thus, we can finally calculate the PDF of $v^{*}(t+d)$. By the same way, the PDF of irradiance at predicted moment also can be solved.

\section{PREDICTION OF PROBABILISTIC POWER FLOW}

\section{A. Latin Hyper-cube Sampling (LHS)}

Monte Carlo sampling technique is often used to calculate the probabilistic power flow. It will get a high calculation precision under the condition that the sample size is large enough. Obviously, it demands amount of calculation.

Latin hyper-cube sampling not only applies stratified sampling technique to ensure that the samples can fully cover the distribution range of the input random variables, but also can eliminate the correlation of multiple random variables by sorting the samples. At present, LHS has been used in reliability calculation of power system [17].

In this paper, LHS is applied to sample wind speed and irradiance according to their PDF. The samples of wind speed and irradiance are separately transferred to wind power and photovoltaic power, which are then substituted into power flow equation to calculate probabilistic power flow of micro-grid.

\section{B. Joint probability Distribution Prediction}

There is no obvious correlation between wind speed and irradiance at same place. Even if there is some correlation, it is hard to find a mathematical model to describe it. Although the correlation of wind speed and irradiance is weak, but the power flow of micro-grid is a function of wind speed and irradiance. At a certain moment in the future, once the distribution interval of wind speed and irradiance are identified, the calculated distribution interval of power flow is identified also. So we propose two calculation methods of probabilistic power flow for micro-grid which are unconditional and conditional joint probability distribution technique.

(a) Unconditional joint probability distribution (UJPD)

Firstly, we apply LHS for the PDF of wind speed and irradiance that are achieved by the approach proposed above. Wind speed and irradiance samples are randomly combined into sample couples on the condition that their weak correlation is ignored, such as $\left\{V_{i}, S_{i}\right\}, i=1, \cdots, n$, where $n$ is sampling size. Then these sample couples are transformed to wind power and photovoltaic power to calculate power flow. Finally, we solve PDF and confidence intervals of node voltage or branch power at predict moment with kernel density estimation method. For example, The PDF of node voltage can be calculated by

$$
f\left(u_{t+d}\right)=\frac{1}{N_{i} h} \sum_{m=1}^{N_{i}} k\left(\frac{u_{t+d}-u_{m}}{h}\right)
$$

Where, $k(x)$ is kernel function, $u_{\mathrm{m}}$ is the sample of node voltage, $h$ is bandwidth coefficient, $N_{\mathrm{i}}$ is total number of samples. In the same way we can also calculate the PDF of active and reactive power.

(b)Conditional Joint Probability Distribution (CJPD)

Unlike UJPD, the intervals $D_{1}$ and $D_{2}$ of predictive wind speed $V_{\mathrm{i}}$ and irradiance $S_{\mathrm{i}}$ are used as constraint conditions to select the sampling subset from all sample couples that meets the conditions $V_{i} \in D_{1}$ and $S_{i} \in D_{2}$.

After the subset samples are converted to wind generation and photovoltaic generation, we can further substituted them into the power flow equations to calculate the samples of power flow. Finally, kernel density estimation method is applied also to calculate the PDF, CDF of node voltage or branch power at predictive moment.

\section{The confidence interval of predictive power flow}

The confidence interval of predictive power flow can be solved based on PDF of power flow achieved by above UJPD and CJPD technique.

Taking node voltage $u$ for example, if parameter $\alpha(0<\alpha<1)$ meet $P\left(u_{\text {min }}^{*}<u<u_{\text {max }}^{*}\right)=1-\alpha$, then the 
interval $\left[u_{\text {min }}^{*}, u_{\text {max }}^{*}\right]$ is called confidence interval of $u$ with confidence coefficient $1-\alpha$, which denotes that the interval comprise the observed value with probability of $1-\alpha$.

According to the PDF of node voltage, we select intervals whose cumulative probability are greater than or equal to confidence level $1-\alpha$. In all eligible intervals, the narrowest one is selected as the confidence interval of predictive node voltage to ensure the precision and credibility.

\section{RESULTS AND VALIDATION}

BenchMark $0.4 \mathrm{kV}$ low-voltage micro-grid as showed in figure 2 is adopted in the paper to verify the proposed approach of probabilistic power flow prediction. There are 20 nodes and 19 branches in the micro-grid system. The line parameters can be found in the literature [18]. We assign that the reference direction of branch power is from the node of small label to the node of large label.

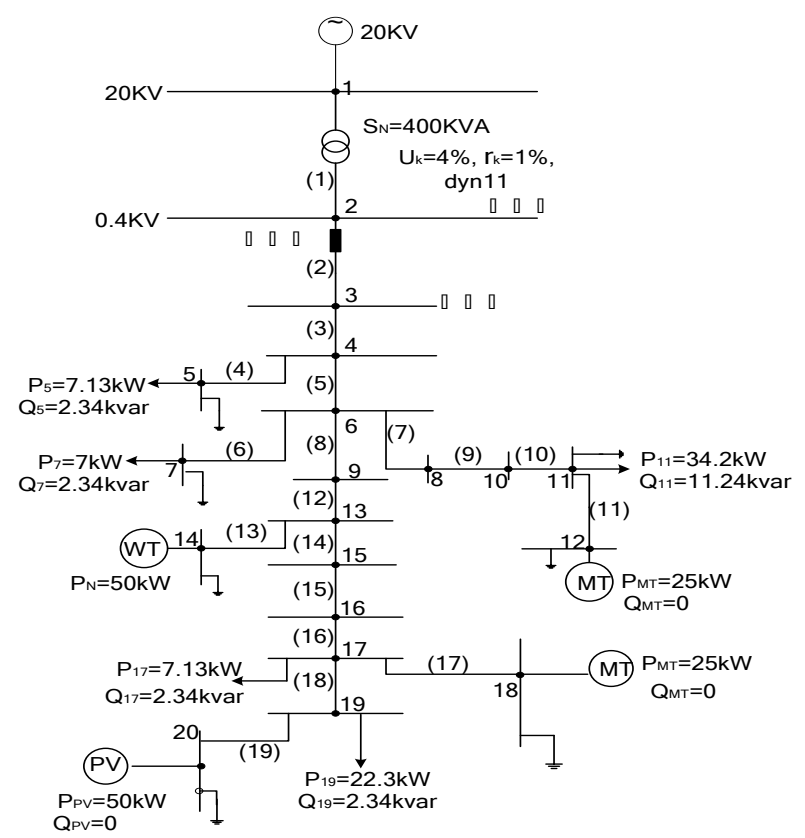

Figure 2. BenchMark $0.4 \mathrm{kV}$ low-voltage micro-grid.

In figure 2, the network parameters are three-phase symmetrical. The WT connected to node 14 is an asynchronous wind power generator whose rated power is $50 \mathrm{~kW}$. The PV connected to node 20 is photovoltaic power station of rated power $50 \mathrm{~kW}$, and the MT connected to node 12 and 18 are gas turbines. The all loads connected to the grid are of constant power. Usually, micro-grid is connected to the power grid through the public access point. In power flow calculation progress, the node 1 connected to power grid is regarded as balance node. Because photovoltaic devices and gas turbine are of voltage control strategy, so they are regarded as PV nodes. On the contrary, asynchronous wind power generator is regarded as PQ node due to it is not of voltage adjustment ability. In following simulation example, Newton-Ralph algorithm is adopted to calculate power flow.

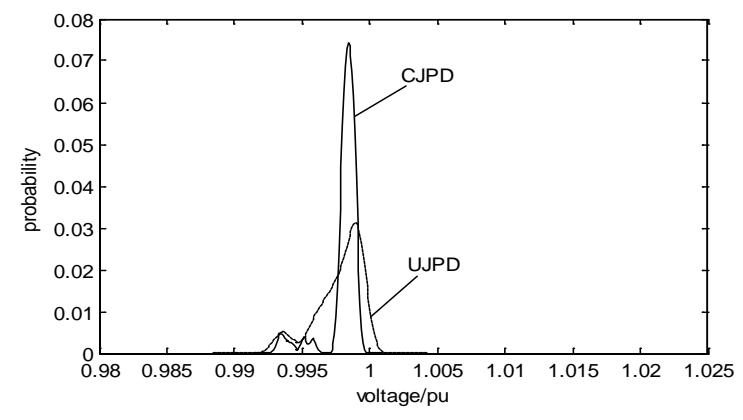

Figure 3. Voltage PDF of node 14 at the 12th hour.

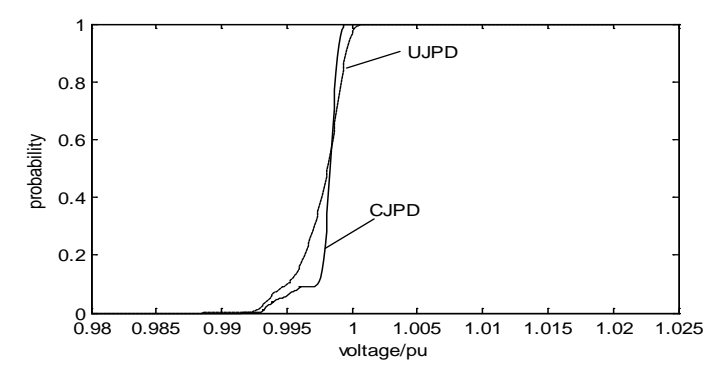

Figure 4. Voltage CDF of node 14 at the 12th hour.

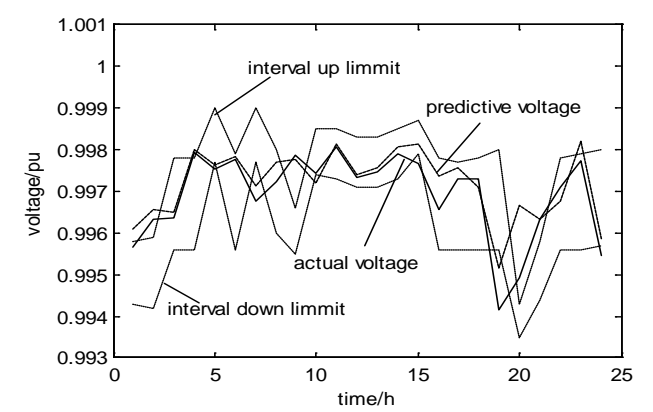

Figure 5. Voltage predictive curves and conditional joint probability intervals of the 14th node.

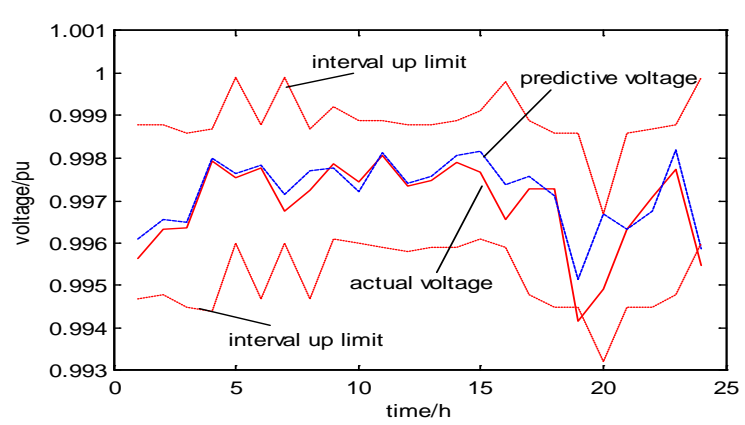

Figure 6. Voltage predictive curves and unconditional joint probability intervals of the 14th node.

Figure 3 and Figure 4 are the PDF and CDF of the 14th node voltage at the 12th hour during a day, which respectively achieved by the technologies of UJPD and CJPD of the. It can be seen that because the sample couples are constrained by the intervals of wind speed and 
illumination, The CJPD is more concentrated than the UJPD. The node voltages achieved by CJPD prediction technique are distributed in the range of $[0.997,1]$, which present lower uncertainty. However, due to this constraint condition is not taken into consideration in UJPD predictive technique, the node voltages are distributed in the relatively large range of [0.992, 1.001], which present higher uncertainty.

Figure 5 and Figure 6 provide voltage predictive curves of the 14th node and probability intervals achieved by CJPD and UJPD technique at confidence level $90 \%$. It is obvious that the range of conditional joint probability interval is narrower than that of unconditional joint probability interval at the same confidence level. We can find that the predicted error of the 14th node at 17th hour is large, and the predicted results of two techniques are obviously different. The predictive value with UJPD technique can fall within the interval at 90\% confidence level, but the predictive value with CJPD technique fall outside the interval at the same confidence level. The comparison shows that the CJPD is stricter than the UJPD in credibility evaluation of predictive results. The proposed CJPD technique can effectively reduce the risk, and provides more apparent reference meaning.

\section{CONCLUSIONS}

The power flow of micro-grid is affected by wind speed and irradiance at the same time. Therefore the research about probabilistic power flow should not only consider the transition characteristics of the wind speed and irradiance themselves, but also regard the intervals of predictive wind speed and irradiance as constraint conditions for screening sample couples. Visibly, the CJPD technique based on above ideas is more in line with the actual in uncertainly analysis of predictive results in micro-grid which containing wind and photovoltaic generations.

\section{ACKNOWLEDGMENT}

The authors gratefully acknowledge the financial support of the natural science foundation of universities of Anhui province (KJ2014A258) and the personnel foundation of Tongling university (2014tlxyrc01).

\section{REFERENCES}

[1] Lasseter R H. The role of distributed energy resources in futureelectric power systems[C]. Energy Systems Seminar, University of Wisconsin, 2006.

[2] Hatziargyriou N D, Dimeas A, Tsikalakis A G, et al.Management of Micro-grids in Market Environment[C].In: International Conference on Future Power Systems.2005.1-7.
[3] Mao Meiqin, Ding Ming, Chang Liuchen, et al. Testbed and Information Integration of EMS for a Micro-grid with Multi-energy Generation Systems[J]. Automation of Electric Power Systems, 2010, 34(1):106-110(in Chinese).

[4] Katiraei F, Iravani M R, Lehn P W. Micro-grid autonomous operation during and subsequent to islanding process[J]. IEEE Trans. on Power Delivery, 2005, 20(1): 248-257.

[5] Liu Yanghua, Wu Zhengqiu, Lin Shunjiang. Research on Unbalanced Three-phase Power Flow Calculation Method in Islanding Micro Grid. Journal of Hunan University, 2009,36(7):36-40,(in Chinese).

[6] Wang Chengshan, Zheng Haifeng, Xie Yinghua.Probabilistic power flow containing distributed generation in distribution system [J]. Automation of Electric Power Systems, 2005, 29(24): 39-44.

[7] Yu Kun, Cao Yijia, Chen Xingying et al. Dynamic probability power flow of district grid containing distributed generation [J]. Proceedings of the CSEE, 2011, 31(1): 20-25 (in Chinese).

[8] A. Bracale, G. Carpinelli, M. Mangoni etc. Wind Power Forecast Methods and Very Short-term Steady-State Analysis of an Electrical Distribution System Electrical Engineering Research Report vol. 1, issue 1

[9] Zhou Songlin, Mao Meiqin, Su Jianhui. Power Flow Forecasting of Micro-grid considering the Randomness of Wind Power [J]. Proceedings of the CSEE, 2013,33(22): 26-34, (in Chinese).

[10] Wang Min. Probabilistic Model of Distribute Power and Its Influence on Power System [D]. Hefei: Hefei University of Technology, 2010.

[11] Mao Mei-qin, Zhou Songlin, Su Jianhui. Short-term Forecasting of Wind Power on Ridgelet Neural Network[J]. Automation of Electric Power System, 2011,35(7): 1-5(in Chinese)

[12] Fu Meiping, Ma Hongwei, Mao Jianrong. Short-term photovoltaic power forecasting based on similar days and least square support vector machine [J]. Power System Protection and Control 2012, 40(16):65-69(in Chinese)

[13] Ding Ming, Wu Xing long, Lu Wei. Three-phase probabilistic power flow calculation in distribution systems with multiple unsymmetrical grid-connected photovoltaic systems[J]. Automation of Electric Power System, 2012,36(16): 47-51(in Chinese)

[14] D. Huang and R. Billinton. Effects of wind power on bulk system adequacy evaluation using the well-being analysis framework. IEEE Transactions of Power Systems, 2009,24(3):1232-1240.

[15] Ding Ming, Xu Ningzhou. A Method to Forecast Short-Term Output Power of Photovoltaic Generation System Based on Markov Chain. Power System Technology, 2011,35(1):152-157.

[16] Liu Yongqing , Liu Dongsheng. An Anomaly Detection Method Based on Markov Model[J]. Computer \& Digital Engineering. 2010, 38(7): 20-23.

[17] Yu Han, Chung Chiyong, Wong Kitpo. A Probabilistic Load Flow Calculation Method with Latin Hypercube Sampling [J]. Automation of Electric Power Systems, 2009,33(21):32-35

[18] K. Rudion, Z. A. Styczynski, N. Hatziargyriou, S. Papathanassiou, etc. Development of Benchmarks for Low and Medium Voltage Distribution Networks with High Penetration of Dispersed Generation[C]. 3rd International Symposium on Modern Electric Power Systems, Wroclaw, 6-8 Sep., 2006. 